\title{
BMJ Open Protocol of a randomised clinical trial to integrate mental health services into primary care for postconflict populations in Northern Sri Lanka (COMGAP-S)
}

\author{
Shannon Doherty (D) , ${ }^{1}$ Giselle Dass, ${ }^{2}$ Anne Edwards, ${ }^{2}$ Robert Stewart, ${ }^{3}$ \\ Bayard Roberts, ${ }^{4}$ Melanie Abas ${ }^{5}$
}

To cite: Doherty S, Dass G, Edwards A, et al. Protocol of a randomised clinical trial to integrate mental health services into primary care for postconflict populations in Northern $\mathrm{Sri}$ Lanka (COMGAP-S). BMJ Open 2022;12:e051441. doi:10.1136/ bmjopen-2021-051441

- Prepublication history for this paper is available online. To view these files, please visit the journal online (http://dx.doi org/10.1136/bmjopen-2021051441).

Received 18 March 2021 Accepted 05 January 2022

\section{Check for updates}

(c) Author(s) (or their employer(s)) 2022. Re-use permitted under CC BY-NC. No commercial re-use. See rights and permissions. Published by BMJ.

${ }^{1}$ School of Allied Health, Faculty of Health, Education, Medicine and Social Care, Anglia Ruskin University, Chelmsford, UK

${ }^{2}$ THEME Institute, Colombo, Sri Lanka

${ }^{3}$ Institute of Psychiatry, King's College London, London, UK

${ }^{4}$ Department of Health Services Research and Policy, London School of Hygiene and Tropical Medicine, London, UK

${ }^{5}$ Section of Epidemiology, Health Services \& Population Research, Institute of Psychiatry, King's College London, London, UK

Correspondence to

Dr Shannon Doherty;

shannon.doherty@anglia.ac.uk

\section{ABSTRACT}

Introduction Sri Lanka has a long history of armed conflict and natural disasters increasing the risk of mental health disorders in the population. Due to a lack of specialist services, there is a treatment gap between those seeking and those able to access mental health services. The aim of this research programme is to integrate mental health services into primary care to meet the needs of this postconflict population.

Methods and analysis This is a stepped wedge cluster design randomised clinical trial of the WHO mental health Gap Action Programme primary care mental health training intervention. We will provide a 10-day training to primary care practitioners of 23 randomly selected primary care facilities aimed at increasing their ability to identify, treat and manage common mental health disorders. Public health professionals and community representatives will receive a tailored training intervention to increase mental health awareness. Refresher courses will occur at 3 and 6 months post training. Supervision and monitoring will occur for 1 month pre and post training. Target sample sizes have been calculated separately for each group of participants and for each outcome.

Ethics and dissemination This trial has received ethical approval from the Faculty of Health, Education, Medicine and Social Care, Anglia Ruskin University, UK (SC/jc/ FMFREP/16/17 076) and from the Faculty of Medicine, University of Jaffna, Sri Lanka (J/ERC/17/81/NDR/0170) and non-engagement approval has been received from the funding body, the Centers for Disease Control and Prevention (2018-015). All participants gave written consent. Dissemination of study results will be completed through publication of academic articles, conference presentations, town hall meetings, written pamphlets in plain language, reports to Ministry of Health and other government organisations and through social media outlets.

Trial registration numbers ISRCTN registry: ISRCTN62598070. SLCTR registration number: SLCTR/2018/008.

\section{INTRODUCTION}

Mental health disorders often present in primary care settings, especially in low-income and middle-income countries (LMIC),
Strengths and limitations of this study

- First study to culturally adapt all WHO mental health Gap Action Programme (mhGAP) written and video training materials.

- Seeks to demonstrate feasibility of using WHO mhGAP materials to train non-specialists.

- Multiple refresher sessions should increase knowledge retention among participants.

- Representative sample means findings could be generalised to other areas of Northern Sri Lanka.

- Limited to publicly available healthcare facilities as private facilities were excluded.

where specialised psychiatric services may be lacking. Low or no detection, low referral rates for specialist care and increased costs are all features of mental health disorders in primary care settings in LMIC. Furthermore, lack of adequate training for primary care physicians and lack of involvement of public health personnel can act as barriers to effective treatment and management of common mental health disorders in primary care. ${ }^{12}$ Barriers can be compounded in postconflict situations, as existing limited health systems may be severely affected, especially for those internally displaced. There is strong evidence that conflict-driven, internally displaced migrants have increased rates of mental health disorders. ${ }^{13}$

During 26 years of protracted internal conflict in Sri Lanka, more than 100000 people of all ethnicities are estimated to have died, and hundreds of thousands injured. ${ }^{45}$ Substantial internal displacement from conflict was compounded by the 2004 tsunami. ${ }^{6}$

Although Sri Lanka has an effective primary care system, the treatment gap for mental health is extensive, especially for postconflict populations. ${ }^{17}$ While provision of health 
services has improved, resources are insufficient to meet population needs, especially those related to severity of trauma, or to difficulties experienced in displacement or return migration. ${ }^{8}$ Primary care practitioners in the Northern Province region regularly spearhead mental healthcare efforts, however, they do so without adequate training. Training primary care practitioners to deliver mental healthcare at primary care level is in line with the task-shifting approach in the global mental health field. ${ }^{2-11}$ A pilot feasibility study (COMGAP) to explore this possibility was conducted in 2013 and 2014 based on a peer-reviewed protocol and is reported elsewhere. ${ }^{8}$

The first phase of the study was completed in 2015 and 2016 and facilitated understanding of mental disorder burden and treatment gap at primary care level. Results from the cross-sectional study indicated the most prevalent mental health disorders in primary care settings were depression $(41.1 \%$, 95\% CI: $38.7 \%$ to $44.5 \%)$, anxiety ( $46.7 \%$, $95 \%$ CI: $41.9 \%$ to $51.5 \%$ ), post-traumatic stress disorder (13.7\%, 95\% CI: $10.6 \%$ to $16.8 \%$ ) and psychosis with hypomania (17.6\%, 95 CI: 13.3 to 21.9$).{ }^{12}$ While this facilitated understanding of the underlying mental health issues within the region, the issue of unmet need was still present. In this context, the aim of phase II of COMGAP-S is to use a scaled-up training intervention based on the WHO Mental Health Gap Action Programme (mhGAP 2.0 ) to integrate mental health services into primary care by providing training to primary care practitioners and public health professionals serving conflict-affected populations in primary care settings in Northern Province. ${ }^{12} 13$ The second phase of the project was conducted between 2016 and 2021. COMGAP-S is the first large-scale trial in the region to use mhGAP to integrate mental health services into primary care for conflict-affected populations. Further, it is the only trial to include wider healthcare staff and community members as part of a long-term strategy to improve mental health awareness and stigma reduction.

\section{Aims and objectives}

The aim of COMGAP-S is to investigate if implementation of WHO mhGAP training within primary care settings increases identification and treatment of common mental disorders for postconflict populations in Northern Province, Sri Lanka.

\section{Primary outcomes}

1. Overall, 30\% increase in patients identified and treated for common mental health disorders and referrals to specialist care for complex mental disorders by primary care practitioners, measured at baseline using the premonitoring/postmonitoring questionnaires on current practice with mental health patients and compared with post-training practice.

2. Overall, $40 \%$ minimum concurrence between diagnoses of patients identified with mental disorders of interest by trained primary care practitioners and psychiatrists as compared with diagnosis of patients pre train- ing (baseline) measured using quantitative reporting forms from the consultant psychiatrist and in-depth, individual interviews with trained primary care practitioners to understand any changes in practice.

3 . Overall, $20 \%$ reduction in positive screening for depression and anxiety in patients pre training and at 3-month and 6-month follow-up time points, measured using the Hopkins Symptom-Checklist 25.

\section{Secondary outcomes}

1. Overall, $20 \%$ increase between mean pretraining and post-training test scores for primary care practitioners using the WHO mhGAP 2.0 pretraining/post-training test.

2. At least $50 \%$ reduction of mental health stigma measured using Attitudes to Mental Illness Questionnaire (AMIQ) in primary care practitioner, public health professional and community representative participant groups at training and 3-month and 6-month follow-up. Prevalence rates were based on previous mental health studies conducted within the country. ${ }^{15}$

3. At least $40 \%$ of primary care practitioners and public health professionals in the region will be delivered training on mhGAP, and at least two community representatives from each facility catchment areas will receive tailored training on mental health awareness and stigma reduction.

\section{METHODS AND ANALYSIS \\ Study design}

This is a randomised clinical trial using a stepped wedge cluster design. ${ }^{14}$ This design comprises a preliminary period where no clusters (healthcare facilities) are exposed to the intervention, then at regular intervals, one cluster is randomised to cross from control to intervention. This continues until all clusters have been exposed..$^{14}$ The stepped wedge design is increasingly used to evaluate interventions that involve service delivery and involves sequential and random crossover of clusters (facilities) from control (delivery of standard care) to intervention (mhGAP training) until all clusters are exposed. ${ }^{14}$ The stepped wedge design takes into account logistical constraints to sequentially roll out the intervention by training one facility at a time enabling us to understand how this intervention can be implemented in the future on a larger scale. This design was chosen because: (1) in an intervention trying to integrate mental health services into primary care for postconflict populations, it is unethical to use a parallel design which prevents equal distribution of knowledge and skills and (2) the stepped wedge cluster design is more logistically feasible in the postconflict setting of Northern Sri Lanka. ${ }^{15}$

Facilities will be randomised using standardised randomisation techniques to allocate facilities to the training sequence within the stepped wedge design. Patients and community representatives will not be randomised. The study team cannot be blinded to the allocation of facilities. 
The study psychiatrist will be masked to randomisation status of facilities from which patients originate. Patients will also be blinded to facility randomisation status. ${ }^{1}$

There is a theoretical possibility of contamination due to close professional or personal networks between participant groups and geographical location of facilities. However, this is not envisaged to exert significant influence on expected outcomes due to specifics of the extensive training, requirement to carry out intensive tasks and low risk of contamination through informal discussions. ${ }^{1}$ Potential contamination will be observed at all stages and taken into account at the end of the study. Further, an important strength of cluster sampling is that it helps mitigate the risk of treatment contamination between intervention and control groups.

\section{Setting}

The study setting will be 23 government primary care health facilities within the five districts of the Northern Province: Jaffna, Mannar, Kilinochchi, Vavuniya and Mullaitivu. A full list of participating sites can be obtained from the primary investigator.

A list of public primary care facilities was provided to the study team by Northern Ministry of Health. A random number generator will be used to select 23 facilities across five districts. Distribution of clusters will be allocated proportionally to total numbers of internally displaced people in each district (districts with larger numbers were assigned more clusters). This will ensure adequate representation of conflict displacement and severity.

\section{Inclusion criteria}

Facilities will be included if they indicate willingness to participate, are located in any of the five districts of Northern Province (Jaffna, Mannar, Kilinochchi, Vavuniya, Mullaitivu), provide primary care services and are either divisional hospitals of type A, B and C or primary medical care units. Facilities should also provide services to conflict-affected, previously displaced populations.

Primary care practitioners will be included if they have full registration with the Sri Lankan Medical Council, have at least 6 months or more until their next transfer rotation or 6 months to retirement. Public health professionals will be included if they have at least 6 months left on their transfer rotation or 6 months to retirement. Community representatives located within catchment areas of each selected facility will be included after identification through local registration organisations. Patients will be included if they are 18 years or older, attend selected facilities and belong to internally displaced or conflict-affected populations.

\section{Exclusion criteria}

Larger facilities such as district hospitals and teaching hospitals will be excluded as they are not part of the primary care system. Private facilities will be excluded due to lack of an official registry.
Primary care practitioners, public health professionals and community representatives will be excluded if they have secondary mental health training. Patients under 18 years and those diagnosed with mental disorders outside of depression and/or anxiety will be excluded.

\section{Recruitment and enrolment}

Support letters from Provincial Department of Health Service and Regional Divisional Health Secretariats in each district of Northern Province will be used to approach primary care practitioners and public health professionals. Community representatives will be recruited through local registration organisations. Patients will be recruited by primary care practitioners. Trained research team members will perform screening and enrolment procedures and gain informed consent for each participant group following established study protocols.

\section{Structure and delivery of the intervention}

Training material was adapted for local use and included translation and cultural adaption of relevant modules of the WHO mhGAP 2.0 IG and production of locally developed mhGAP video material for the Tamil context. ${ }^{16}$ This was completed in collaboration with local academics, psychiatrists, primary care practitioners, Northern Province Ministry of Health and the University of Jaffna.

Training will consist of delivery of written materials, role plays and training videos. All training material was developed in collaboration with Sri Lankan researchers, local psychiatrists and community physicians. All material was translated, back translated and piloted for cultural appropriateness prior to the full project.

Almost all instruments used in the study had been previously applied in epidemiological studies within Sri Lanka and are available in Tamil, Sinhala and English languages. New instruments were translated, back translated and adapted for cultural appropriateness in collaboration with the Sri Lankan research team. Further, the entire set of measures was field tested in a pilot study prior to the full study commencing. This pilot study examined if measures were understood by participants, culturally acceptable and translations correct.

To ensure safety of participants, a local psychiatrist will be involved throughout the study to provide free mental healthcare if required.

\section{Primary care practitioners and patients}

Primary care practitioners (general physicians practicing in selected facilities) will undergo 1 month of monitoring prior to training to establish a baseline. Primary care practitioners (PCP) will be trained using the mhGAP manual to recognise common mental health disorders using screening procedures, deliver brief psychosocial interventions (psychoeducation, motivational interviewing) and for referral procedures for complex cases. They will receive a paper booklet to record information on patients identified, treated, managed and/or referred for mental health disorders. Further, before training, primary care 
practitioners will be asked to recruit up to eight patients using provided inclusion and exclusion criteria to have anxiety and/or depression diagnoses verified by a psychiatrist. Members of the research team will provide patients with information sheets and consent forms available in all three official languages (English, Tamil, Sinhala) and obtain informed consent. Patients will complete a brief sociodemographic questionnaire and the Hopkins Symptom Checklist 25 screening questionnaire for depression and anxiety at the point of recruitment, and at 3 and 6 months, follow-up will be conducted to establish a baseline and determine if patient outcomes change after training of primary care practitioners. Primary care practitioners will be asked detailed questions about their management and treatment of recruited patients, and patients will be asked about any treatments undertaken or medications prescribed to understand any intervening factors between baseline and 3-month and 6-month follow-up.

After the 1-month monitoring period, primary care practitioners will undergo a 3-day training intervention delivered in their primary care facility. Trained members of the research team will deliver training at each selected facility to minimise disruption of work at facilities. PCP trainers have master's degrees in psychology and global mental health and have successfully completed the WHO mhGAP train the trainers programme.
After completion of training, monitoring of primary care practitioners will continue for 1 month, where they will continue to use the paper booklet to record information on patients they identify, treat and manage, for common mental health disorders and any cases they refer to specialised care. Research team members will visit or telephone the primary care practitioners once a week at their facility to monitor implementation of the training intervention.

After the 1-month monitoring period, primary care practitioners will continue to use paper booklets to record their experience of the training implementation for 6 weeks. At this point ( 3 months post training), primary care practitioners will participate in a 1-day refresher course and continue to use the experience of training implementation application for a further 3 months. At 6 months post training, primary care practitioners will take a second 1-day refresher course (figure 1).

Non-clinical supervision at all time points will be provided by research assistants who previously delivered training sessions. Supervision will consist of phone calls or visits to the clinics to understand if and how training is being used by participants. Supervision is meant to understand if and how trained participants use mhGAP, accurate delivery of interviewing or diagnostic skills were not evaluated.

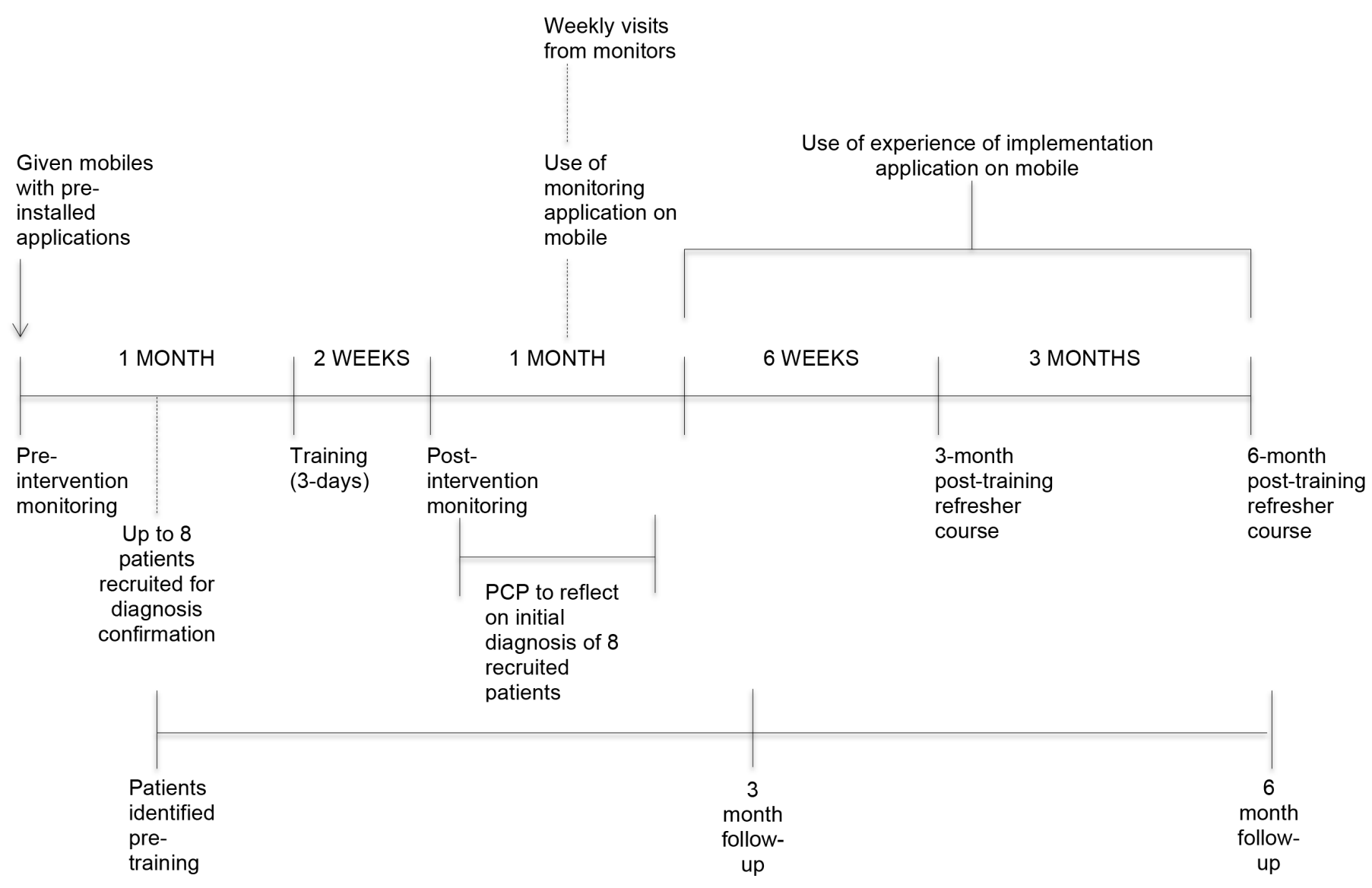

Figure 1 Primary care practitioner study timeline. 


\section{Public health professionals}

Public health professionals (nurse attendants, midwives) in each selected facility will undergo 1 month of monitoring prior to training to establish a baseline. Public health professionals (PHP) will be trained using adapted written materials from the mhGAP training to recognise signs of common mental health issues, provide basic psychosocial care (education) and refer onwards if needed. PHP trainers hold bachelor's degrees in psychology and have undergone 7-day training with senior members of the research team. Each enrolled public health professional will record information in a paper booklet on facilitation of referral and follow-up practices for mental health patients and support primary care practitioners with patient management activities. After the 1-month monitoring period, public health professionals will be provided with a tailored 3-day training programme on mental health awareness, management, referral and stigma reduction. After training, public health professionals will undergo 1 month of monitoring and record the same information as in the premonitoring period to see how/if practice has changed. Research team members will visit or telephone their facility once a week to monitor implementation of the training intervention.

After the 1-month monitoring period, public health professionals will record information on their experience of the training intervention for 6 weeks. At this point (3 months post training), public health professionals will participate in a 1-day refresher course and continue to use the paper booklets to record their experience of training implementation for a further 3 months. At 6 months post training, public health professionals will take a second 1-day refresher course (figure 2).

Non-clinical supervision at all time points will be provided by research assistants who delivered previous training sessions. Supervision will consist of phone calls or visits to the clinics to understand if and how training was being used by participants.

\section{Community representatives}

Community representatives (teachers, social workers) will undergo 1 month of monitoring prior to training to establish a baseline. Community representatives (CR) will be trained using adapted materials from the mhGAP training material to recognise signs of common mental illness, deliver basic psychosocial education and learn referral pathways and where to seek providers. CR trainers hold bachelor's degrees in psychology and have undergone 7-day training with senior members of the research team. Each enrolled community representative will record information in a paper booklet on mental health awareness raising activities undertaken and any referrals of people they encounter with mental health issues. After the 1-month monitoring period, community representatives will participate in a tailored 1-day training programme on mental health awareness, stigma reduction, and finding local resources. After training, community representatives will undergo 1 month of monitoring and record the same information as in the premonitoring period to see how/if practice has changed. Research team members will visit or telephone once a week to monitor implementation of the training intervention.

After the 1-month monitoring period, community representatives will record information on their experience of the training intervention in paper booklets for 6 weeks. At this point ( 3 months post training), community representatives will participate in a half-day refresher course and continue to use provided booklets to record their experience of training implementation for a further 3 months. At 6 months post training, community representatives will participate in a second half-day refresher course (figure 3 ).

Non-clinical supervision at all time points will be provided by research assistants who delivered previous training sessions. Supervision will consist of phone calls or visits to the clinics to understand if and how training was being used by participants.

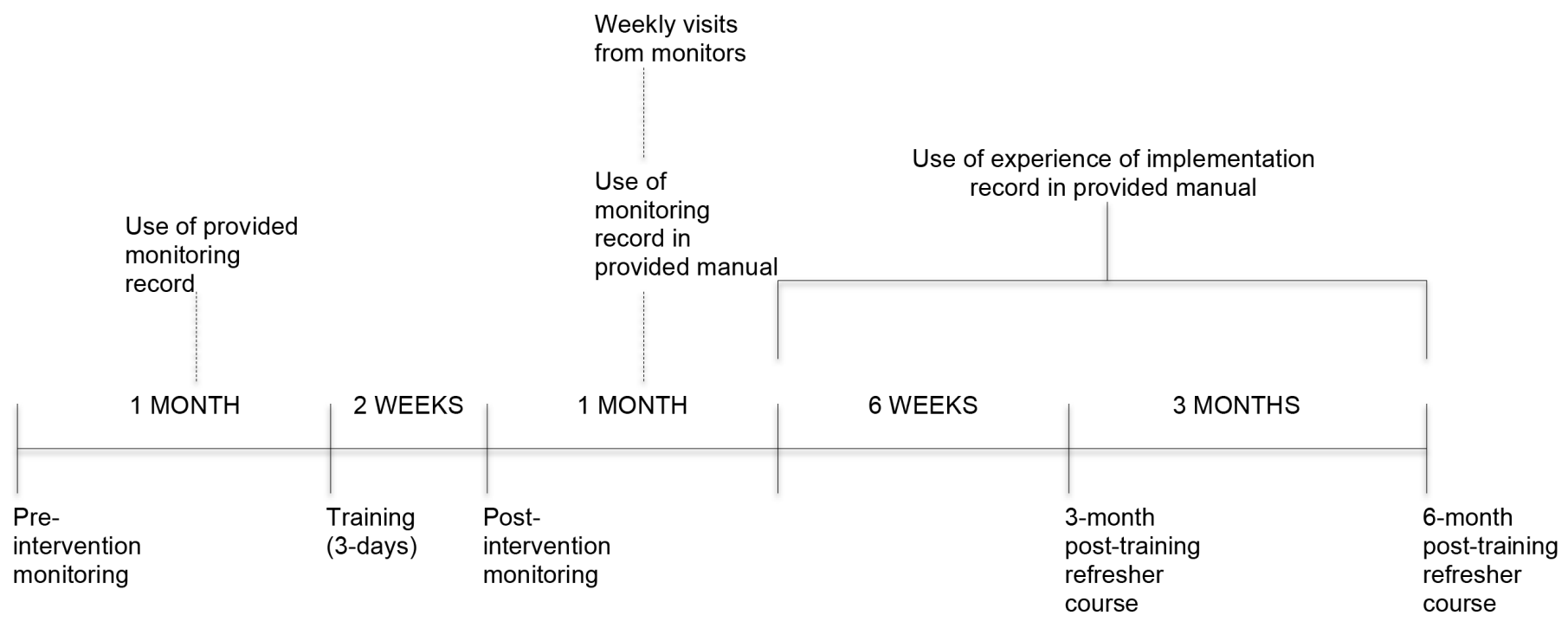

Figure 2 Public health professional study timeline. 


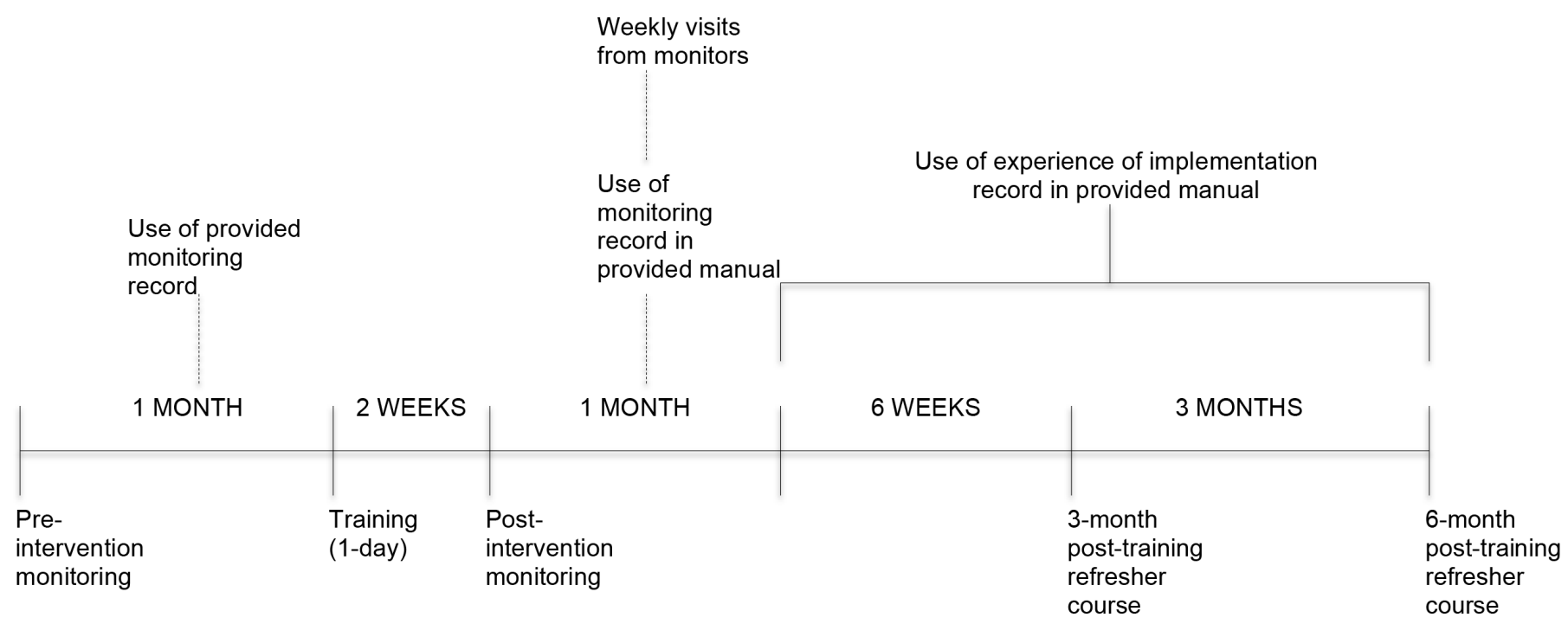

Figure 3 Community representative study timeline.

\section{Timing of intervention delivery and follow-up}

COMGAP-S will run for 7 months in each facility and catchment area. Every 2 weeks a facility will be enrolled in the study until all 23 have completed the study timeline. Participants discontinuing engagement will not be subject to follow-up. Measures will be collected at six time points:

1. Pretraining monitoring period: questionnaire to establish baseline on current practice. Primary care practitioners only: recruitment of patients for diagnosis verification, patients administered Hopkins Symptom Checklist 25 for anxiety and/or depression (baseline), at 3-month and 6-month follow-up to assess change in clinical symptoms.

2. Pretraining intervention: WHO mhGAP knowledge pretest and AMIQ stigma questionnaire.

3. Post-training intervention: second administration of WHO mhGAP knowledge test.

4. Post-training monitoring period: questionnaire to establish if/how practice has changed, and qualitative interviews to reflect on initial diagnosis practice.

5. Three-month follow-up training: WHO mhGAP pre and post knowledge test and AMIQ stigma questionnaire.

6. Six-month follow-up training: WHO mhGAP pre and post knowledge test and AMIQ stigma questionnaire.

\section{Statistical methods}

The primary unit of analysis (ie, where the intervention is carried out) is the healthcare facility, and data collected from individual participants will include: sociodemographic information, conflict and displacement experience, mhGAP knowledge test scores, AMIQ stigma scores and premonitoring and postmonitoring questionnaires. Descriptive statistics will be used to summarise observations using means, SD and proportions where appropriate. Data analysis will be conducted using SAS V.9.4M $7 .^{17}$
An intention-to-treat analysis will be used to analyse the effect of the intervention. In the stepped wedge design, similar information will be collected repeatedly on individuals within facilities; we will therefore use statistical models that can adjust for repeat measures and clustering of participants within facilities (in the case of PHPs and CRs). In particular, for the analysis of mhGAP knowledge test scores and AMIQ stigma scores as outcomes of interest among participants, we plan to use generalised linear mixed models that include random intercepts for individuals (to account for repeat measurements), random intercepts for facilities (to account for the correlation among individuals within the same facility) and adjustments for covariates. Statistical analysis will be carefully conducted as repeated time measures over a 7-month time period will mean missing data points and shifted timelines due to external circumstances. Subgroup analysis will be conducted to understand differences between clusters and well as within clusters. Planned subgroup analysis will investigate possible differences between clusters (eg, differences between PHPs across facilities).

For qualitative data, interviews with primary care practitioners will continue until saturation has been met and thematic analysis will be used.

Fidelity checks will be included through utilisation of feedback forms after the end of each training session to investigate if any major concerns have arisen that could be addressed before subsequent sessions. Further, a process evaluation will be conducted to understand if the study adhered to protocol and how the intervention was received by participants.

\section{Sample size calculation}

A total of 23 primary care practitioners, 75 public health professionals, 50 community representatives and 200 patients will be recruited. Target sample sizes were calculated separately for each participant group and for each outcome. Sample sizes for the first and second outcomes 
were calculated following methods described by Hemming and Taljaard, where each outcome is considered an individually randomised trial and then multiplied by a design effect to account for the stepped wedge design. ${ }^{18}$ Sample sizes for the third and fourth outcomes were calculated using formulae from the 1994 textbook 'Analysis of longitudinal data' by Diggle et al. ${ }^{19}$

\section{Ethics and dissemination}

Ethical approval has been received from the Faculty of Health, Education, Medicine and Social Care, Anglia Ruskin University, UK (SC/jc/FMFREP/16/17 076) and the Faculty of Medicine, University of Jaffna, Sri Lanka (J/ERC/17/81/NDR/0170) and non-engagement approval has been received from the funding body, the Centers for Disease Control and Prevention (2018-015). Trial results and analyses will be purposefully communicated to the Provincial Department of Health Service and Regional Divisional Health Secretariats in all five districts of Northern Province, participating healthcare professionals and community representatives. Dissemination events will be held within the University of Jaffna and the wider community. Activities will include publications and presentations to general and specialised audience by team members. The research dataset and statistical analyses will be available by request from the primary investigator.

\section{Evaluation}

Cost ratio economic evaluation

This component will involve two evaluations. The first will evaluate the ratio between costs associated of training primary care practitioners to identify, treat and refer mental healthcare patients compared with costs of referral to and treatment from psychiatrists. Costs will be linked to the primary outcome measure to determine the extra cost of training incurred to detect one extra patient and understand economic implications of service provision to conflict-affected populations. ${ }^{20}$ The second will evaluate the ratio between costs associated with patients receiving care from trained primary care practitioners at primary care facilities compared with costs of seeking care from specialist psychiatrists.

\section{Process evaluation}

After initial training, 3-month and 6-month refresher courses, quantitative and qualitative feedback will be collected from participants to explore how training was received. Qualitative case studies with primary healthcare staff, trainers, supervisors and patients will take place during post-training monitoring and evaluation periods to understand how the programme was received and implemented. Descriptive analysis will be used for quantitative data, while thematic analysis will be used for qualitative data.

\section{DISCUSSION}

Due to the history of conflict and displacement in Sri Lanka, it is vital to address unmet mental health needs.
The completed cross-sectional survey at primary care level (phase I) supports this and demonstrates prevalence and predictors of mental health disorders in the region. The WHO mhGAP IG 2.0 training programme provides a strategy to address this gap between those seeking and those able to access mental health services in the region (phase II). COMGAP-S will not only demonstrate the feasibility of implementing the mhGAP training programme, but ongoing monitoring activities will contribute to evaluation of both training and implementation.

Using the mhGAP guide to train participants to identify and treat common mental health disorders could empower primary care practitioners to effectively address mental health needs of the population in this postconflict setting. This will also take pressure off of limited specialised psychiatry services in the region. Additionally, training public health professionals and community representatives can positively increase mental health awareness and decrease stigma in the local community. Use of a locally adapted mhGAP guide will increase acceptability and sustainability of the implementation of the clinical trial. This project includes key stakeholders from Northern Ministry of Health, University of Jaffna, Sri Lankan psychiatrists and Sri Lankan researchers. Thus, COMGAP-S will not only contribute to the evidence base on integrating mental health services in primary care in low-resource settings but it will also build local capacity, be culturally relevant and sustainable.

COMGAP-S will be the first large-scale implementation of WHO mhGAP IG 2.0 training to improve mental health service delivery in primary care in Northern Province, Sri Lanka. Implementation of COMGAP-S aims to not only build capacity within the primary care system in the region but also to improve mental health service delivery addressing the unmet needs of a vulnerable population.

\section{Twitter Melanie Abas @melanieabas}

Acknowledgements The authors would like to sincerely thank Dr Chesmal Siriwardhana, who unexpectedly passed away in 2016. Dr Siriwardhana was the original principal investigator and was responsible for the initial conception of this study.

Collaborators Not applicable.

Contributors SD developed the concept and design of this study and wrote the manuscript with contributions from GD, AE, BR, RS and MA. All authors commented on and approved the final version of this manuscript.

Funding This work was supported by the Centers for Disease Control and Prevention, Cooperative Agreement Number, 1U01GH001654-01. The Funding organization was involved in consultations regarding the scientific validity of the design, analysis of the data, preparation and review of the manuscript for publication. The Northern Ministry of Health, Sri Lanka was involved in management of the data collection. The University of Jaffna, Sri Lanka was involved in consultations on the design of the study, collection and interpretation of data, preparation and review of the manuscript for publication.RS is part-funded by: (i) the National Institute for Health Research (NIHR) Biomedical Research Centre at the South London and Maudsley NHS Foundation Trust and King's College London; (ii) a Medical Research Council (MRC) Mental Health Data Pathfinder Award to King's College London; (iii) an NIHR Senior Investigator Award.

Competing interests RS declares research support received in the last 5 years from Roche, Janssen, GSK and Takeda. 
Patient and public involvement Patients and/or the public were involved in the design, or conduct, or reporting, or dissemination plans of this research. Refer to the Methods and analysis section for further details.

Patient consent for publication Not applicable.

Provenance and peer review Not commissioned; externally peer reviewed.

Open access This is an open access article distributed in accordance with the Creative Commons Attribution Non Commercial (CC BY-NC 4.0) license, which permits others to distribute, remix, adapt, build upon this work non-commercially, and license their derivative works on different terms, provided the original work is properly cited, appropriate credit is given, any changes made indicated, and the use is non-commercial. See: http://creativecommons.org/licenses/by-nc/4.0/.

ORCID iD

Shannon Doherty http://orcid.org/0000-0002-6123-1238

\section{REFERENCES}

1 Siriwardhana C, Adikari A, Pannala G, et al. Prolonged internal displacement and common mental disorders in Sri Lanka: the COMRAID study. PLoS One 2013;8:e64742.

2 Patel V, Belkin GS, Chockalingam A, et al. Grand challenges: integrating mental health services into priority health care platforms. PLoS Med 2013;10:e1001448.

3 Makhashvili N, Chikovani I, McKee M, et al. Mental disorders and their association with disability among internally displaced persons and returnees in Georgia. J Trauma Stress 2014;27:509-18.

4 Somasundaram D. Recent disasters in Sri Lanka: lessons learned. Psychiatr Clin North Am 2013;36:321-38.

5 Husain F, Anderson M, Lopes Cardozo B, et al. Prevalence of warrelated mental health conditions and association with displacement status in postwar Jaffna district, Sri Lanka. JAMA 2011;306:522-31.

6 Siriwardhana C. Windows of opportunity after a disaster: the case of Sri Lanka. Asian Bioethics Review 2010;2.

7 Jenkins R, Baingana F, Ahmad R, et al. Social, economic, human rights and political challenges to global mental health. Ment Health Fam Med 2011;8:87-96.
8 Siriwardhana C, Adikari A, Pannala G. Changes in mental health prevalence among long-term displaced and returnee forced migrants in Sri Lanka (COMRAID-R). BMC Psych 2015;15:41.

9 Patel V, Chowdhary N, Rahman A, et al. Improving access to psychological treatments: lessons from developing countries. Behav Res Ther 2011;49:523-8.

10 Chibanda D, Mesu P, Kajawu L, et al. Problem-Solving therapy for depression and common mental disorders in Zimbabwe: piloting a task-shifting primary mental health care intervention in a population with a high prevalence of people living with HIV. BMC Public Health 2011;11.

11 Lund C, Tomlinson M, De Silva M, et al. PRIME: a programme to reduce the treatment gap for mental disorders in five low- and middle-income countries. PLoS Med 2012;9:e1001359.

12 Doherty S, Hulland E, Lopes-Cardozo B, et al. Prevalence of mental disorders and epidemiological associations in post-conflict primary care attendees: a cross-sectional study in the Northern Province of Sri Lanka. BMC Psychiatry 2019;19:83.

13 World Health Organization. mhGAP training manuals for the mhGAP intervention guide for mental, neurological and substance use disorders in non-specialized health settings, 2017. Available: https:// www.who.int/publications-detail/mhgap-intervention-guide-version2.0

14 Brown CA, Lilford RJ. The stepped wedge trial design: a systematic review. BMC Med Res Methodol 2006;6:54.

15 Siriwardhana C, Adikari A, Jayaweera K, et al. Integrating mental health into primary care for post-conflict populations: a pilot study. Int J Ment Health Syst 2016;10:12.

16 Doherty S, Dass G, Edward A, et al. Challenges and lessons learned in re-filming the WHO mhGAP training videos for Sri Lankan context - a qualitative study. Confl Health 2020;14:7.

17 SAS Institute. v9.4M6. Cary, NC: 2018.

18 Hemming K, Taljaard M. Sample size calculations for stepped wedge and cluster randomised trials: a unified approach. $J$ Clin Epidemiol 2016;69:137-46.

19 Diggle P, Diggle PJ, Heagerty P. Analysis of longitudinal data. Oxford University Press, 2002.

20 Chisholm D, James S, Sekar K, et al. Integration of mental health care into primary care. Br J Psychiatry 2000;176:581-8. 